\title{
Zwanzig Jahre Makroökonomischer Dialog der EU
}

\section{Neustart für den Euroraum und die Mitgliedstaaten}

Der Makroökonomische Dialog der EU besteht nunmehr seit zwanzig Jahren. Verlief im ersten Jahrzehnt seines Bestehens die gesamtwirtschaftliche Entwicklung über den gesamten Euroraum gesehen durchaus befriedigend, so zeigten die zwischenzeitlich aufgelaufenen massiven gesamtwirtschaftlichen Divergenzen zwischen den Mitgliedstaaten und die dadurch verursachte Krise, dass es einer vertieften Institutionalisierung der makroökonomischen Koordinierung bedarf. Sie muss insbesondere einer stärkeren Abstimmung zwischen Euroraum und Mitgliedstaaten Rechnung tragen. Hierzu wird die Einrichtung eines Makroökonomischen Dialogs sowohl für den Euroraum als auch auf nationaler Ebene und deren Umsetzung unter deutscher Präsidentschaft im zweiten Halbjahr 2020 im Rahmen der umfassenden Economic Governance Review der EU-Kommission vorgeschlagen.

Am 1.1.1999 wurde die Europäische Wirtschafts- und Währungsunion (WWU) gegründet. Es sollte eine Wirtschaftsund eine Währungsunion zugleich sein, also weder erst Währungsunion als Zugpferd für die Wirtschaftsunion („Lokomotiv-Theorie“) noch der „krönende“ Abschluss der Wirtschaftsunion durch die Währungsunion („Krönungstheorie“), sondern beides in einem und sich gegenseitig verstärkend. Räumlich wie politisch umfasste sie zwei Elemente: den Euroraum als Ganzen und die einzelnen Mitgliedstaaten. Die Aufmerksamkeit richtete sich dabei lange primär auf das neue Element: den Euroraum mit dem Euro als gemeinsamer Währung und ohne nationale Wechselkurse. Der Euro sollte stabil wie die D-Mark sein. Preisstabilität erwartete man sich von der Stabilität der öffentlichen Finanzen, obwohl der Konnex zwischen Defiziten und Inflation alles andere als gesichert ist. ${ }^{1}$ Der Stabilitäts- und Wachstumspakt (SWP) sollte diese Stabilisierung unter Begrenzung von Defizit und Schuldenstand gewährleisten.

Die Erfahrung gerade in Deutschland hätte aber eine andere, zumindest zusätzliche Vorkehrung nahegelegt. Die beiden Ölpreiskrisen nach 1973 und 1980 hatten eine (Öl-) Preis-Lohn-Preisspirale verursacht, die von der Geldpolitik jedes Mal mit massiver Restriktion bekämpft wurde. Das

(c) Der/die Autor(en) 2020. Open Access: Dieser Artikel wird unter der Creative Commons Namensnennung 4.0 International Lizenz (http:// creativecommons.org/licenses/by/4.0/deed.de) veröffentlicht.

Open Access wird durch die ZBW - Leibniz-Informationszentrum Wirtschaft gefördert.

1 „Fiscal discipline has always been considered a necessary prerequisite for the orderly functioning of a monetary union geared towards price stability.", European Fiscal Board: Assessment of EU fiscal rules with a focus on the six- and two-pack legislation, August 2019, S. 8.
Ergebnis waren gleichzeitig Inflation und ein schockartiger Anstieg der Arbeitslosigkeit. ${ }^{2}$ Projiziert auf den Euroraum bedeutete das, derartige Konflikte zwischen den makroökonomischen Akteuren zu vermeiden und - positiv gewandt - für einen zugleich wachstums-, beschäftigungs- und stabilitätsorientierten makroökonomischen Policy-Mix durch Kooperation statt Konfrontation zu sorgen.

\section{Die Geburt des Makroökonomischen Dialogs der EU}

Erste Überlegungen ${ }^{3}$ zu einem kooperativen Ansatz wurden im Dezember 1998 von der Präsidentschaft des Europäischen Rats von Wien angestellt: „... ein breiter und intensiver Dialog zwischen allen betroffenen Akteuren, d.h. Rat, Kommission, Europäisches Parlament, Sozialpartner, Euro-

2 Der positive Wiedervereinigungs-,,Schock“ in Deutschland hatte ebenfalls zu einem massiven Konflikt zwischen Lohn- und Geldpolitik, dieses Mal auch unter Beteiligung der (expansiven) Fiskalpolitik geführt.

3 Zu Entstehung und Intentionen des Makroökonomischen Dialogs vgl. W. Koll: Makroökonomischer Dialog - Entstehung und Intentionen, in: E. Hein, T. Niechoj, T. Schulten, A. Truger: Europas Wirtschaft gestalten, Makroökonomische Koordinierung und die Rolle der Gewerkschaften, Hamburg 2004, S. $128 \mathrm{ff}$.

Dr. Willi Koll war Ministerialdirigent in den Grundsatzabteilungen des Bundeswirtschafts- und -finanzministeriums. Zugleich war er langjähriges Mitglied im Wirtschaftspolitischen Ausschuss der EU und der OECD sowie im Makroökonomischen Dialog der EU. 
päische Zentralbank und Europäische Investitionsbank ..." Unabhängigkeit und Autonomie der Beteiligten erforderten eine indikative, „weiche“, statt imperative, „harte“, Koordinierung, also Dialog statt bindender Beschlüsse. Die Offenheit des Dialogs verlangte zudem Vertraulichkeit, auch um gegenseitiges Vertrauen aufzubauen. In diesen Selbstbeschränkungen lagen von Anfang an Stärke und Schwäche des Vorhabens. ${ }^{4}$

Institutionalisiert wurde er auf dem Europäischen Rat von Köln Mitte 1999 durch den Beschluss über die Einrichtung eines „Makroökonomischen Dialogs der EU“, EUMED oder einfach MED. Sein Mandat besteht darin, Lage und Perspektiven der gesamtwirtschaftlichen Entwicklung zu bewerten, die daraus folgenden wirtschaftspolitischen Herausforderungen zu identifizieren und den Beitrag aller Akteure zu deren Bewältigung zu diskutieren. Richtschnur waren die "Grundzüge der Wirtschaftspolitik der Mitgliedstaaten und der Gemeinschaft". Teilnehmer des MED waren anfänglich eine "Troika“ aus vergangenem, aktuellem - als Vorsitz - und zukünftigem Präsident des Rats „Wirtschaft und Finanzen“ (ECOFIN) sowie die entsprechenden Arbeitsminister, die Kommissare für Wirtschaft und Währung sowie für Beschäftigung, der Präsident der EZB und ein Vertreter der Präsidenten der Nicht-WWU-Zentralbanken sowie die europäischen Spitzen der Sozialpartner, d. h. Vertreter der Arbeitgeber aus Industrie, kleinen und mittleren Unternehmen und öffentliche Arbeitgeber sowie der Europäische Gewerkschaftsbund. Ursprünglich waren auch die Vorsitzenden von Wirtschafts- und Finanzausschuss, Wirtschaftspolitischem Ausschuss und Beschäftigungsausschuss beteiligt; sie wurden jedoch später nicht mehr einbezogen. Der Dialog auf politischer Ebene wird durch eine jeweils ganztägige Sitzung auf Fachebene vorbereitet. Der Vorsitz informiert das Europäische Parlament über die Ergebnisse der Treffen. Die erste Runde des MED auf fachlicher und politischer Ebene fand im Herbst 1999, die bisher letzte Runde im November 2019 in Brüssel statt.

\section{Performance des Makroökonomischen Dialogs der EU}

Nimmt man die ersten Jahre der WWU als Maßstab, so ist die Entwicklung des Euroraums als Ganzem zumindest nicht gegen die erklärte Intention des MED verlaufen. Wachstum, Beschäftigung und Preisstabilität wurden zugleich erreicht. Sicherlich hat das gegenseitige "Signalisieren“ während

4 Kritiker des MED sehen in diesem Arrangement eher eine Schwäche. Dagegen befindet Engels unter Verweis auf die Europa-Forschung „..., dass die geringe Verbindlichkeit und der informelle Rahmen der Gesprächsrunde dem Europäischen Makroökonomischen Dialog nicht zum Nachteil gereichen, sondern ganz im Gegenteil politikbezogenes Lernen und die Vertrauensbildung zwischen den makroökonomischen Akteuren begünstigen können.“ Vgl. J. Engels: Die Krise als Chance? - Gegenwärtige Barrieren und mögliche Anknüpfungspunkte für eine Koordinierung der Geld-, Lohn- und Fiskalpolitik in der Europäischen Währungsunion, Marburg 2016, S. 417. und zwischen den Sitzungen mit dazu beigetragen, dass die politischen Akteure ihre souveränen Entscheidungen auch im Lichte der Signale der jeweils anderen Partner getroffen haben. ${ }^{5}$

Zunächst waren die Diskussionen im MED primär auf das Zusammenspiel von Geldpolitik und WWU-weiter Lohnentwicklung gerichtet. Konkret stand den Forderungen der Gewerkschaften nach möglichst niedrigen Zinsen für mehr Beschäftigung die Forderung der EZB nach stabilitätsgerechten Löhnen - jedenfalls über den Durchschnitt des Euroraums gesehen - gegenüber. Auch für die Arbeitgebervertreter spielten die Zinsen eine Rolle, da sie aus ihrer Sicht insbesondere auch den Außenwert des Euro und damit die preisliche Wettbewerbsfähigkeit der Unternehmen beeinflussen. Ein entscheidender Mangel war, dass - anders als in der Eurogruppe - nicht alle Finanzminister, sondern nur die oben genannte Troika präsent war. Dieser Mangel wurde auch nicht dadurch geheilt, dass nach einigen Jahren auch der Vorsitzende der Eurogruppe am MED teilnahm. So war die Fiskalpolitik unterrepräsentiert und blieb auf ECOFIN und Eurogruppe beschränkt. Die EZB wiederum war lange nur an der Entwicklung der Eurozone als Ganzer interessiert. Dadurch aber fehlt dem MED die Kopplung und Rückkopplung zur nationalen Ebene, d.h. zur wirtschaftlichen Entwicklung in und zwischen den Mitgliedstaaten. Dieses Defizit war schon in einer Bilanz nach dem ersten Jahrzehnt des MED bemängelt worden. So wurde eine verbesserte $A b-$ stimmung zwischen EU und EU-Mitgliedstaaten ebenso gefordert wie die Ergänzung des MED um ein eurozonenspezifisches Element (EUROMED). ${ }^{6}$ Konzeptionell ging es um eine stärkere Beachtung und Überwachung lohnpolitischer Orientierungsmarken, d.h. der Orientierung der Lohnstückkosten am Preisziel der EZB in jedem Mitgliedstaat, wie von den „Grundzügen der Wirtschaftspolitik für die Mitgliedstaaten und die Gemeinschaft" eingefordert, um die weitere Verstärkung der bereits massiv ausgeprägten Divergenzen zu

5 Vgl. V. Hallwirth, W. Koll: Zehn Jahre Makroökonomischer Dialog - eine Zwischenbilanz, in: Wirtschaftsdienst, 89. Jg. (2009), H. 1, S. 26-33; V. Hallwirth, A. Watt: The policy mix and policy coordination in EMU how can it contribute to higher growth and employment?, in: Transfer, European Review of Labour and Research, 9. Jg. (2003), H. 4.

6 Vgl. V. Hallwirth, W. Koll, a.a.O., S. 32 ff.; vgl. auch insbesondere zu den Divergenzen W. Koll, V. Hallwirth: Macro Matters: Der Makroökonomische Dialog der Europäischen Union, in: WSI Mitteilungen, 62. Jg. (2009), H. 9, S. 470 f. Die Forderung nach einem Makroökonomischen Dialog für den Euroraum und einer besseren Abstimmung mit der nationalen Ebene wurde später immer wieder erhoben. Vgl. hierzu z.B. Europäischer Wirtschafts- und Sozialausschuss: ECO/286 „Makroökonomische Ungleichgewichte“, Brüssel, 5.5.2011; Wirtschaftskammer Österreich: Europa in der globalen Welt absichern, Forderungskatalog der WKÖ an das Europäische Parlament und die Europäische Kommission für die Periode 2014 bis 2019, März 2015; Europäischer Gewerkschaftsbund: Exekutivausschuss, Einbindung der Gewerkschaften in das Europäische Semester und verstärkter Kapazitätsaufbau im Bereich sozialer Dialog und Arbeitsbeziehungen (Entschließungsentwurf), Brüssel 16./17.12.2015. 
stoppen. Ferner sollte die Fiskalpolitik stärker einbezogen werden. Auch die EU-Kommission betonte in ihrer ZehnJahres-Bilanz des Euroraums eine stärkere Verantwortung der nationalen Wirtschaftspolitik und deren Thematisierung im MED. ${ }^{7}$ Der Deutsche Gewerkschaftsbund (DGB) forderte schon 2004 für den Europäischen Verfassungskonvent die Verankerung des MED im (damaligen) EG-Vertrag. ${ }^{8}$ Zur „Entlastung“ des MED muss gesagt werden, dass er seit seinem Beginn den EU-kontinentalen wissenschaftlichen Mainstream und den dadurch beeinflussten wirtschaftspolitischen Konsens gegen sich hatte, der unter Verkürzung der makroökonomischen Dimension primär auf eine Strukturreformagenda fixiert war. ${ }^{9}$

\section{Krise und Anpassung im Euroraum}

Seit Beginn der WWU entwickelten sich unterhalb der Ebene des Euroraums massive Divergenzen in der Lohn- und Preisentwicklung und damit bei Realzins, Binnennachfrage, Wettbewerbsfähigkeit sowie im Ergebnis bei Leistungsund Kapitalbilanz. Ursache war eine gleichzeitig permanente, persistente und sich selbstverstärkende Prozyklik von Fiskalpolitik sowie von Lohn- und Preisentwicklung in den Mitgliedstaaten:10 im „Süden“ eine starke, das Preisziel der EZB überschießende, im „Norden“ eine schwache, das Preisziel unterschreitende Entwicklung. ${ }^{11}$ Wer wie viele nur auf den Durchschnitt im Euroraum schaute, sah indes keinen Anlass zur Sorge. So blieben auch im MED diese Divergenzen (zu) lange unbeachtet. Warnungen und Vorschläge hatte es allerdings genug gegeben. ${ }^{12}$ Sie blieben folgenlos. Als sie die Beachtung fanden, die sie verdienten, war es zu spät. Im Gefolge der globalen Finanzkrise entluden sich die inzwischen massiven Divergenzen schockartig in einem „sudden stop“ der Waren- und Finanzströme.

7 „It seems necessary to increase ownership of national stakeholders by stimulating a debate in the context of the Macroeconomic Dialogue (MED)“. Vgl. European Commission: EMU@10 - Successes and challenges after 10 years of Economic and Monetary Union, in: European Economy, Nr. 2, 2008, S. 291.

8 Vgl. Deutscher Gewerkschaftsbund: Ein neuer Sozialkontrakt für Europa, Empfehlungen des Deutschen Gewerkschaftsbundes an den Europäischen Konvent, in: E. Hein, T. Niechoj, T. Schulten, A. Truger: Europas Wirtschaft gestalten, Makroökonomische Koordinierung und die Rolle der Gewerkschaften, Hamburg 2004, S. 240 ff.

9 Vgl. W. Koll, V. Hallwirth, a. a. O., S. 469.

10 Vgl. W. Koll, A. Watt: Vertiefung und Konvergenz der Europäischen Wirtschafts- und Währungsunion durch konzeptionelle und institutionelle Reformen der makroökonomischen Koordinierung, IMK, Study Nr. 61, Juni 2018, Hans-Böckler-Stiftung, Düsseldorf 2018.

11 Eine Ausnahme war Frankreich mit einer ausgewogenen Entwicklung im Einklang mit dem Preisziel der EZB.

12 Vgl. hierzu unter anderem „Verpasste Chancen“, in: W. Koll: Neue Wirtschaftsregierung und Tarifautonomie in der Europäischen Union - Makroökonomische Koordinierung im Dialog, IMK, Study 30, Düsseldorf, März 2013, S. 34 ff. So wurde bereits 2006 aus dem Makrodialog auf fachlicher Ebene heraus ein Vorschlag für einen MED für den Euroraum vorgelegt, der aber leider auf politischer Ebene nicht die Zustimmung aller Beteiligten des MED fand.
Die wenigen Instrumente, die länderspezifisch ausgerichtet waren, wie an erster Stelle der Stabilitäts- und Wachstumspakt (SWP), konnten dieses Ergebnis nicht verhindern. Der SWP und spätere Fiskalregeln wie z.B. der Fiskalpakt wirkten eher restriktiv, wo mehr Flexibilität angebracht gewesen wäre. Auch zwischenzeitliche Reformen des Pakts reichten nicht aus, erhöhten aber seine Komplexität. ${ }^{13}$ Erst spät reifte die Erkenntnis, dass die Fokussierung auf Fiskalaspekte in einer WWU viel zu kurz greift. ${ }^{14}$ Diese Fixierung auf den öffentlichen Sektor verstellte zudem den Blick dafür, dass die Kumulation von Ungleichgewichten primär und zuerst im privaten Sektor stattfand.

Allzu lange wertete man die inflatorischen Tendenzen in Südeuropa als ein erwünschtes Aufholen. Allerdings blieb dort die inländische Wertschöpfung in jedem Jahr deutlich hinter der Binnennachfrage zurück. Dies wäre weniger problematisch gewesen, wäre das importierte Kapital in potenzialsteigernde Investitionen geflossen. In Nordeuropa brachten die eher deflatorischen Tendenzen Deutschland den Befund struktureller Sklerose und den Titel „Kranker Mann Europas" ein, anstatt hier schlicht makroökonomische Expansion einzufordern und umzusetzen.

\section{Die Krise als Wendepunkt}

Die Krise des Euroraums war ein tiefer Einschnitt und Wendepunkt. Einige der Defizitländer wurden dem Anpassungsprogramm der Troika unterworfen. Rezession, Sozialabbau und ein schockartiger Anstieg der Arbeitslosigkeit waren die Folgen. Aus makroökonomischer Sicht verlief die Bewältigung der Eurokrise alles andere als optimal: in den Defizitländern eine massive Schrumpfung, ohne eine expansive Gegenbewegung in den Überschussländern, damit im Euroraum insgesamt zu wenig Dynamik und ein starker Leistungsbilanzüberschuss gegenüber dem Rest der Welt: rezessive Anpassung anstelle von expansiver Korrektur. ${ }^{15}$

13 Vgl. European Fiscal Board, a.a. O

14 Vgl. European Commission: Directorate-General for Economic and Financial Affairs, in: Quarterly Report on the Euro Area, 9. Jg. (2010), H. 1, S. 6 (vgl. auch dort S. 38: Beschluss der Eurogruppe). „Es war ein Fehler, dass wir vor allem auf die Staatsfinanzen geschaut haben. Wir hätten ein breiteres Spektrum makroökonomischer Parameter überwachen sollen... Die Entwicklung der Lohnstückkosten, Leistungsbilanzdefizite, Immobilienblasen. Die Kommission hätte ein Mandat gebraucht, gegen diese Ungleichgewichte genauso vorzugehen wie gegen zu hohe Staatsverschuldung.“, vgl. K. Regling: Interview „Rettungsfonds - Der Bund verdient eine ordentliche Marge“, in: Berliner Zeitung vom 23./24.11.2010, S. 2.

15 „High-debt countries would commit to reduce their debt, and symmetrically low-debt countries would commit to increase growth-enhancing government expenditure, in particular those that have positive cross-border spillovers. The proposed agreement would effectively implement a euro area aggregate fiscal stance. Finally, the creation of links between net expenditure growth and the MIP could be explored." European Fiscal Board, a.a. O., S. 7. 
Die EZB ging - nicht zuletzt wegen einer in einigen Mitgliedstaaten sogar stark restriktiven Fiskalpolitik - zu einer extrem expansiven Geldpolitik über, was den Defizitländern eine allmähliche Erholung ermöglichte. Die Überschussländer erhielten hierdurch ebenfalls einen Impuls, der in einigen zinsreagiblen Bereichen wie der Bauwirtschaft in Deutschland fast schon eine Überhitzung brachte und die Immobilienpreise sprunghaft ansteigen ließ, im Euroraum insgesamt aber relativ begrenzt ausfiel, wie nicht zuletzt die dauerhaft niedrige Preisinflation zeigt. Auf den Finanzmärkten trieb die Niedrigzinspolitik die Aktienkurse auf neue Höchststände.

Zur Verbesserung der Economic Governance des Euroraums waren im Anschluss an die Krise zahlreiche neue Regelungen und Institutionen eingeführt worden. Es gab vielfältige neue Vorschläge, Verfahren und Berichte. Die meisten davon waren indes fiskalischer oder finanzmarktpolitischer Art. So beinhalteten das Six- und Twopack mit der Verordnung zur Vermeidung und Korrektur gesamtwirtschaftlicher Ungleichgewichte (einschließlich Durchsetzungsverordnung) nur ein Verfahren, das auf die Ungleichgewichte zwischen den Mitgliedstaaten gerichtet war. Aber auch das Verfahren - so wichtig seine Zielsetzung - ist bis heute mit gravierenden Mängeln behaftet - Asymmetrie (d.h. einseitiger Fokus auf Defizitländer), Korrektur statt Prävention, zu schwache Sanktionen, um nur einige wesentliche zu nennen. ${ }^{16}$ Dass die Bankenunion vorangekommen ist, ist erfreulich und notwendig, aber bei weitem nicht hinreichend auf dem Weg zu einer besseren Economic Governance des Euroraums.

\section{Neustart der WWU - Diagnose und Therapie}

Dem vorherrschenden wirtschaftswissenschaftlichen $\mathrm{Pa}$ radigma und seiner Umsetzung durch die Politik mangelt es an einer tragfähigen und umfassenden Diagnose des Entstehens der Krise und einer ebenso tragfähigen Therapie zu ihrer Bewältigung.

\section{Als Diagnose ist festzuhalten: ${ }^{17}$}

- Die schädlichen Divergenzen und die Krise des Euroraums sind aufgrund einer permanent und persistent wirkenden Prozyklik der Wirtschaftsentwicklung und der gesamten makroökonomischen Politik unter Einfluss der Fiskalregeln in vielen Mitgliedstaaten entstanden.

\footnotetext{
16 European Fiscal Board, a. a. O., S. 53.
}

17 Vgl. hierzu ausführlich W. Koll, A. Watt, a.a. O.; W. Koll, A. Watt: Konvergenz von Lohnentwicklung und Makropolitik für eine stabile Europäische Wirtschafts- und Währungsunion, in: WSI Mitteilungen, 72. Jg. (2019), H. 2, S. $117 \mathrm{ff}$.
- Fehlgeleitete wissenschaftliche Politikberatung und mangelnde Einsicht der makroökonomischen Akteure auf nationaler Ebene standen am Anfang dieses Prozesses und begleiteten inn bis zur Krise.

- Auf der Ebene der Eurozone war dieses Versagen nicht auszugleichen und nach der Krise nur um den Preis einer massiven Dehnung der Geldpolitik in seinen Folgen lediglich abzumildern.

Die Therapie ergibt sich konsequenterweise wie folgt:

- Identifikation von makroökonomischen Regeln für die Mitgliedschaft in einer Wirtschafts- und Währungsunion;

- insbesondere Einhaltung der sogenannten „Goldenen Regel" für die nationale Lohn-(Stückkosten-) und Preisentwicklung, d. h. deren Orientierung am Preisziel der Geldpolitik;18

- möglichst günstige monetäre Bedingungen für Investitionen und Beschäftigung; ${ }^{19}$

- Ausbau der Verordnung zur Vermeidung und Korrektur gesamtwirtschaftlicher Ungleichgewichte (VMU) zu einer umfassenden makroökonomischen Benchmark unter Fokussierung auf präventive Elemente wie z.B. auf die obengenannte „Goldene Regel“;20

- Reform des SWP, Stärkung seiner wechselseitigen Verbindung zur VMU und Integration beider Regeln in den makroökonomischen Zusammenhang; ${ }^{21}$

18 So auch L. A. Pereira da Silva, B. Mojon: Exiting low inflation traps by "consensus": nominal wages and price stability, Based on the keynote speech at the Eighth High-level Policy Dialogue between the Eurosystem and Latin American Central Banks, Cartagena de Indias, Kolumbien, 28.-29. November 2019, S. 15. „What is needed are better guidelines for wage and price evolution, ..." O. Blanchard: The Missing Third Leg of the Euro Architecture: National Wage Negotiations, Peterson Institute for International Economics, 28.2.2018. Vor allem Flassbeck hat seit Beginn der WWU immer wieder gefordert, dass diese Regel, die schon Anfang der 1960er Jahre von Meinhold aufgestellt wurde, „... im Zentrum aller makroökonomischer Überlegungen stehen sollte, ..... Vgl. H. Flassbeck, C. Lapavitsas: Nur Deutschland kann den Euro retten, Der letzte Akt beginnt, Frankfurt a. M. 2015, S. 31.

19 Schon unmittelbar vor Beginn der WWU forderten die „Policy Guidelines“: „Je stärker die Geldpolitik bei ihrer Aufgabe, die Preisstabilität zu wahren, von einer angemessenen Haushaltspolitik und Lohnentwicklung unterstützt wird, umso größer ist die Aussicht auf wachstums- und beschäftigungsfreundliche monetäre Rahmenbedingungen.“ European Commission: 1998 Broad Economic Policy Guidelines, European Economy, Nr. 66, Brüssel 1998.

20 Vgl. hierzu European Fiscal Board, a.a. O., S. 53.

$21, \ldots$...econcile stabilisation better with sustainability ..." and „... linking the MIP with fiscal surveillance.", vgl. ebenda, S. 7 und S. 55. Neben vielen anderen Verbesserungen schlägt der EFB insbesondere eine Ausgabenregel als Benchmark für den SWP und eine Variante der Goldenen Regel für öffentliche Investitionen vor. 
- regelmäßige Analyse und Bewertung von Lage und Perspektiven der nationalen makroökonomischen Entwicklung vor dem Hintergrund des Regelwerks;

- regelkonforme Ausrichtung der gesamten makroökonomischen Politik;

- Fokussierung der Nationalen Reformprogramme und der Länderspezifischen Empfehlungen auf den makroökomischen Policy-Mix, d.h. das Zusammenspiel von Fiskalpolitik und Lohnentwicklung unter der einheitlichen Geld- und Währungspolitik;

- Berücksichtigung struktureller Reformen, wenn sie starke Auswirkungen auf die makroökonomische Entwicklung haben bzw. letztere auf sie stark zurückwirkt (z.B. Auswirkungen der Digitalisierung oder die Erreichung sozial- und umweltpolitischer Ziele); ${ }^{22}$

- Abstimmung der nationalen gesamtwirtschaftlichen Politik mit einer zielführenden Politikausrichtung auf der Ebene des Euroraums;

- gegebenenfalls "subsidiäre“ makroökonomische Stabilisierung bzw. Korrektur verbleibender Fehlentwicklungen der nationalen Wirtschaftsentwicklung durch Instrumente auf der Ebene des Euroraums;

- wirksame Sanktionierung bei Nichtbefolgung der Regeln. ${ }^{23}$

\section{Institutionalisierung und Umsetzung}

Auch der MED hat im zweiten Jahrzehnt seines Bestehens Verbesserungen erfahren. So liegt nunmehr der Vorsitz im für den MED zuständigen Wirtschaftspolitischen Ausschuss der EU und im vorbereitenden MED auf fachlicher Ebene in einer Hand. Ferner wurden die Vorbereitung durch eine schriftliche Berichterstattung über die Ergebnisse des MED auf fachlicher Ebene und ein Kernpunktepapier der Ratspräsidentschaft verbessert, der Verlauf durch die Vorgabe eines Spezialthemas weiter strukturiert und die wesentlichen Stellungnahmen der Teilnehmer (mit Ausnahme der EZB) veröffentlicht.

22 Vgl. W. Koll: Makroökonomische Aspekte der Digitalisierung, in: Wirtschaftsdienst, 99. Jg. (2019), H. 1, S. 41-46; vgl. z. B. derselbe: Vom Stabilitäts- und Wachstumsgesetz zum Wohlstands- und Nachhaltigkeitsgesetz, in: Wirtschaftsdienst, 96. Jg. (2016), H. 1, S. 40-48.

23 So wurde bisher kein Verfahren wegen übermäßigen Ungleichgewichts eingeleitet. „Making access to funds from a potential central fiscal capacity conditional upon compliance with the EU fiscal rules could improve compliance." Vgl. ebenda, S. 7 und S. 81.
Diese hier skizzierten Verbesserungen bewegen sich im Rahmen der bisherigen „Architektur“ des MED. ${ }^{24}$ Die speziellen Herausforderungen der WWU erfordern indes neue Strukturen der Koordination und Kooperation. Zu deren Institutionalisierung wird vorgeschlagen: ${ }^{25}$ Einrichtung eines unabhängigen makroökonomischen Beratungsgremiums in jedem Mitgliedstaat und für den Euroraum; Einrichtung eines makroökonomischen Dialogs in jedem Mitgliedstaat (MEDNAT); Einrichtung eines makroökonomischen Dialogs für den Euroraum (EUROMED) ${ }^{26}$. Die Institutionalisierung sollte wie folgt umgesetzt werden:

1. First best: Einrichtung neuer Gremien: Einrichtung eines unabhängigen Beratungsgremiums sowohl für den Euroraum als auch auf nationaler Ebene. Seine Aufgaben sind die gesamtwirtschaftliche Analyse und die Erarbeitung von möglichst quantifizierten gesamtwirtschaftlichen Szenarien und wirtschaftspolitischen Optionen für einen zugleich wachstums-, beschäftigungs- und stabilitätsorientierten makroökonomischen Policy-Mix. ${ }^{27}$ Teilnehmer des MEDNAT sollten sein: der Finanzminister (als Vorsitz und Mitglied von ECOFIN, Eurogruppe und EUROMED), der Wirtschafts- und der Arbeitsminister, das nationale Mitglied des ESZB, die Spitzenvertreter der Sozialpartner und der Berichterstatter des Beratungsgremiums. Die Treffen sollten auf Fachebene inhaltlich vorbereitet werden, deren Ergebnisse können eine Diskussionsgrundlage im politischen Gremium sein. Eine ausführliche Berichterstattung an das nationale Parlament ist vorzusehen. ${ }^{28}$ Teilnehmer des EUROMED sollten sein: der Präsident der Eurogruppe als permanenter Vorsitz ${ }^{29}$, die Finanzminister aller Mitgliedstaaten der WWU, die Kommissare für Wirtschaft und Währung sowie für Arbeit, der Präsident

24 Für eine umfassende Untersuchung des bisherigen Makroökonomischen Dialogs auf der Basis theoretischer Grundlagen makroökonomischer Koordinierung vgl. J. Engels, a.a. O.

25 Vgl. W. Koll, A. Watt: Vertiefung und Konvergenz ..., a. a. O.; W. Koll, A Watt: Konvergenz von Lohnentwicklung ..., a.a. O., S. $121 \mathrm{ff}$.

26 Ein Makroökonomischer Dialog auf der Ebene des Euroraums ist nicht zuletzt auch im Bericht der fünf Präsidenten vorgeschlagen worden. Vgl. J.-C. Juncker, D. Tusk, J. Dijsselbloem, M. Draghi, M. Schulz: Die Wirtschafts- und Währungsunion vollenden, Bericht der fünf Präsidenten vom 22.6.2015, Anhang 2, S. 24. Auch der EWSA hat sich für einen Makrodialog des Euroraums ausgesprochen. Vgl. G. Bischoff, A\&W blog, Technokratie versus Demokratie, 3.11.2015.

27 Angesichts unterschiedlicher ökonomischer Paradigmen der makroökonomischen Akteure über das „wahre“ ökonomische Modell sollen diese Gremien auch dazu beitragen, dass eine gemeinsame Sichtweise über die wechselseitigen Interdependenzen, die wirtschaftspolitischen Herausforderungen und ihre Bewältigung entwickelt wird. Vgl. hierzu J. Engels, a.a. O., Kapitel 7, S. 265 ff.; und A. Heise: Der Kölner Prozess. Theoretische Grundlagen und erste Erfahrungen mit dem EU-Makrodialog, in: Integration, 24. Jg. (2001), H. 4, S. 398.

28 In Deutschland wurde schon zeitgleich mit dem MED 1999 ein nationaler MED auf fachlicher Ebene eingerichtet.

29 Der halbjährliche Wechsel im Vorsitz des MED aufgrund des halbjährlichen Wechsels im Vorsitzes des ECOFIN hat sicherlich nicht zur Kontinuität des MED beigetragen. 
der EZB, die europäischen Spitzenvertreter der Sozialpartner und der Berichterstatter des Beratergremiums. Dabei sollten sich die Sozialpartner - jeder für sich möglichst weitgehend mit ihren nationalen Mitgliedern vorab koordinieren. ${ }^{30}$ Eine ausführliche Berichterstattung an die nationalen Parlamente ist vorzusehen. ${ }^{31}$ Die Vorbereitung der Treffen sollte durch ein fachliches Gremium in der gleichen Zusammensetzung mit anschließender Berichterstattung im politischen Gremium erfolgen. Entscheidend für die Wirksamkeit sowohl des MEDNAT als auch des EUROMED ist die Einbettung beider Gremien in den Ablauf des Europäischen Semesters. Zurecht wird kritisiert, dass sich der bisherige MED außerhalb des Verfahrens- und Entscheidungsverlaufs des Europäischen Semesters bewegt. ${ }^{32}$ Ein Vorschlag zur Integration von EUROMED und MEDNAT wurde an anderer Stelle gemacht. ${ }^{33}$

\section{Second best: Aufbau auf bestehenden Institutionen: Zu} prüfen ist, inwieweit die Agenda bereits bestehender Gremien personell und inhaltlich auf die makroökonomische Agenda ohne Verlust an Effektivität und gegebenenfalls unter Entlastung von bisherigen Aufgaben erweitert werden kann. Als „Kandidaten“ kommen in Betracht: die Nationalen Ausschüsse für Produktivität oder die Fiscal Councils/Independent Fiscal Institutions als nationales Beratergremium; der Europäische Fiskalausschuss als Beratungsgremium auf der Ebene des Euroraums; die Beteiligten bei der Erarbeitung des Nationalen Reformprogramms ${ }^{34}$ und bei der Umsetzung der Länderspezifischen Empfehlungen als MEDNAT; die Eurogruppe, z.B. in Form einer „Extended Informal Eurogroup“ zweimal jährlich, als EUROMED unter Vorbereitung durch die Eurogruppe des Wirt-

30 Vgl. dazu T. Schulten: Gewerkschaftliche Lohn- und Tarifpolitik in Europa - Ansätze, Widerstände und Perspektiven einer europaweiten Koordinierung, in: E. Hein, T. Niechoj, T. Schulten, A. Truger, a. a. O., S. 192 ff.; W. Koll: Neue Wirtschaftsregierung ..., a.a. O., S. 33 ff. und S. 62 ff., T. Müller: European Coordination of collective wage bargaining in times of crisis, in: H. Hein, J. Priewe, A. Watt: Saving the Euro - Redesigning Euro Area economic governance, SE Publishing 2017, S. 371; R. Janssen: Coordinating wages in the European Monetary Union - practical experiences and lessons to be learnt, in: H. Hein, J. Priewe, A. Watt: Saving the Euro - Redesigning Euro Area economic governance, SE Publishing 2017, S. 301; und W. Koll, A. Watt: Warum die Sozialpartner stärker an der wirtschaftspolitischen Koordinierung des Euroraums teilnehmen müssen, in: spw: Europa anders machen - Sozialunion statt Marktunion!, H. 230, 1-2019, S. 45 ff.; vor allem auch Europäischer Gewerkschaftsbund, a.a. O.; und J. Engels, a.a.O., S. $357 \mathrm{ff}$.

31 Zu erwägen ist, den Vorsitz des Wirtschafts- und Währungsausschusses des Europäischen Parlaments direkt am EUROMED zu beteiligen.

32 Engels fordert konsequenterweise als wichtigen Reformschritt, den MED aus dieser „Isolation“ zu lösen. Vgl. J. Engels, a. a. O., S 427.

33 S. Koll, A. Watt: Convergence and Stability ..., a.a. O., S. 23.

34 In Deutschland geht dem die Erstellung des Jahreswirtschaftsberichts der Bundesregierung voran, unter Beteiligung aller makroökonomischen Akteure, bisher allerdings nicht simultan "an einem Tisch“, sondern konsekutiv, d.h. in verschiedenen Gremien. schafts- und Finanzausschusses. Auch beim Aufbau auf bestehenden Institutionen müssen MEDNAT und EUROMED in das Europäische Semester zeit- und aufgabengerecht integriert werden.

Die hier vorgeschlagene kooperative Konzeption und Institutionalisierung beschreiben die Voraussetzung für eine gleichgewichtige Entwicklung in Euroraum und Mitgliedstaaten. Angesichts des noch vorhandenen Anpassungsbedarfs im Gefolge der Krise und der "Schieflage“ im makroökonomischen Policy-Mix - extrem expansive Geldpolitik und zurückhaltende Fiskal- und Lohnpolitik muss dieser zunächst wieder in ein Gleichgewicht seiner Instrumente gebracht werden.

Mit Blick auf die aktuelle Entwicklung schlagen zwei hochrangige Mitglieder der Bank für Internationalen Zahlungsausgleich zur Überwindung der derzeitigen „low inflation traps“ sogenannte „consensus packages“ vor. Nötig sei hierzu eine Kooperation von Geld-, Fiskal- und Lohnpolitik, wie sie sich insbesondere in den 1980er Jahren zur Disinflationierung in Israel, Frankreich und Italien bewährt habe, nun mit dem Ziel der Reinflationierung. Insbesondere soll dadurch die bisherige Überdehnung der Geldpolitik - mit zunehmend geringerem Wirkungsgrad und steigenden negativen Nebenwirkungen - zur Erreichung ihres Preisstabilitätsziels durch eine deutliche Lohnsteigerung und expansive Fiskalpolitik abgelöst werden. Für die Mitglieder der WWU fordern die Autoren eine Koordinierung dieser Politikausrichtung auf der Ebene des Euroraums, um Divergenzen zu vermeiden. Regierungen und Notenbanken sollen diesen Prozess unterstützen bzw. initiieren. ${ }^{35}$ Unabhängig von der derzeitigen konkreten Ausrichtung stützen im Ergebnis Vorschlag und Begründung von „consensus packages“ nach Konzeption und Institutionalisierung den hier beschriebenen „Neustart“ für Eurozone und Mitgliedstaaten.

\section{Die Agenda}

Aufgabe und Tagesordnung für jedes Gremium könnten wie folgt aussehen: ${ }^{36}$

\section{Makroökonomischer Dialog auf nationaler Ebene (MED-} NAT): Diskussion alternativer, vom nationalen Beratungsgremium skizzierter gesamtwirtschaftlicher Entwicklungspfade; Identifikation der daraus folgenden gesamtwirtschaftlichen Erfordernisse; Beiträge der nationalen Fiskal- und Lohnpolitik und gegebenenfalls der makroprudenziellen Politik der nationalen Zentralbank - unter Berücksichtigung der geld- und wäh-

35 Vgl. L. A. Pereira da Silva, B. Mojon, a.a. O.

36 Vgl. W. Koll, A. Watt: Vertiefung und Konvergenz ..., a. a. O., S. 39 ff. 
rungspolitischen Ausrichtung auf WWU-Ebene -, um eine optimale gesamtwirtschaftliche Entwicklung im Einklang mit den Regeln für eine WWU zu erreichen.

Insbesondere sind aus nationaler Sicht zu erörtern: konjunkturelle und strukturelle Ausrichtung der Fiskalpolitik unter Einhaltung des (zu überarbeitenden) SWP und unter Abstimmung mit der fiskalischen Ausrichtung im gesamten Euroraum; Orientierungen zur lohnpolitischen Entwicklung; Überprüfung der gesamtwirtschaftlichen Preisentwicklung und Wettbewerbsintensität; Auswirkung der Einkommens- und Vermögensentwicklung auf die gesamtwirtschaftliche Entwicklung - und umgekehrt; Einhaltung der (zu überarbeitenden) VMU; Umsetzung der länderspezifischen Empfehlungen im makroökonomischen Gesamtzusammenhang; gegebenenfalls stabilitätsgefährdende Entwicklungen auf Finanz- und Assetmärkten.

\section{Makroökonomischer Dialog für den Euroraum (EURO-} MED): Die Agenda des EUROMED beinhaltet - wie die des MEDNAT - einen „Dreiklang“, wie er durch die Entschließung des Europäischen Rats von Köln im Juni 1999 festgelegt wurde: Erörterung der gesamtwirtschaftlichen Lage und Perspektiven im Euroraum anhand des Berichts des hier vorgeschlagenen Beratungsgremiums für den Euroraum und der darin enthaltenen Szenarien und Politikalternativen; Identifikation des wirtschaftspolitischen Handlungsbedarfs, um einen nachhaltig spannungsfreien wachstums- und stabilitätsorientierten makroökonomischen Policy-Mix von Geld- (und Währungs-), Fiskal- und Lohnpolitik im Euroraum zu erreichen und zu wahren; Benennung des eigenen Beitrags und der Erwartungen an den Beitrag der jeweils anderen makroökonomischen Akteure zur Erreichung dieses Zieles; Prüfung der nationalen makroökonomischen Politikausrichtung der Mitgliedstaaten unter anderem anhand der Nationalen Reform- und Stabilitätsprogramme auf Kompatibilität mit den Anforderungen des Euroraums, insbesondere mit dem (überarbeiteten) SWP und der (novellierten) VMU; entsprechende Formulierung der Länderspezifischen Empfehlungen; bessere Sichtbarkeit und Information für die (Finanz-) Märkte durch Veröffentlichung nicht-kontroverser Beratungsergebnisse; Prüfung der Rolle des Europäischen Stabilisierungsmechanismus (ESM) und, soweit neue Elemente wie eine makroökonomische Stabilisierungsfazilität in die Economic Governance eingeführt werden, deren zielführenden Umsetzung; Einbezug des Europäischen Parlaments, zumindest durch ausführliche Unterrichtung.

Zur Umsetzung der Agenda muss jedem Gremium ausreichend Zeit gegeben werden. Der MED kann sein Ziel nur dann erreichen, wenn er sich nicht auf einleitende Stellungnahmen aller Akteure beschränkt, sondern sich daran ein substanzieller Austausch, eben ein „Dialog“ anschließt.

\section{Fazit}

Der Makroökonomische Dialog der EU besteht nunmehr seit zwanzig Jahren. Für die EU als Ganze konzipiert, hat er zugleich auch auf die WWU gewirkt. Bis zur Krise hat es keinen größeren Konflikt zwischen Geldpolitik der EZB und der Lohn- und Preisentwicklung im Aggregat des Euroraums gegeben. Leider kam dieses für sich positive Ergebnis je länger, desto mehr unter Ausbildung von massiven Divergenzen zwischen den Mitgliedstaaten zustande, die sich in einer massiven Krise entluden. Vermieden hätte diese Entwicklung nur eine gleichgewichtige wirtschaftliche Entwicklung unter Orientierung am Preisziel der EZB in jedem Mitgliedstaat. Hierfür wären Verantwortung, Kooperation und Institutionalisierung auf nationaler Ebene seit Beginn der WWU erforderlich gewesen, ebenso ein stärkeres makroökonomisch orientiertes präventives Monitoring und eine Abstimmung auf der Ebene der WWU durch zielführende Gremien. Der MED war dafür in seiner Zusammensetzung und Instrumentalisierung nicht geeignet. Es fehlte die Präsenz eines jeden Mitgliedstaats. Aber auch die Eurogruppe war nicht das angemessene Gremium. Sie war primär mit fiskalpolitischen Themen befasst; um die Preis- und Lohnentwicklung angemessen einzubeziehen, fehlten hier die Sozialpartner.

Seit der Krise geht es vor allem um die Wiederherstellung einer ausgewogenen „Lastenteilung“ zwischen Geld- und Fiskalpolitik. Jedoch greift eine solche Betrachtung zu kurz, weil erneut die makroökonomische Rolle der Löhne vernachlässigt wird. Löhne sind aber eben, je nach Organisationsgrad der Tarifvertragsparteien, nicht nur Getriebene, sondern auch Treiber der gesamtwirtschaftlichen Entwicklung. Im Ergebnis sind die Lohnstückkosten, wie die von ihnen beeinflusste hausgemachte Preisentwicklung, im Zehnjahreszeitraum 2009 bis 2018 im Euroraum jahresdurchschnittlich gerade einmal um rund $1 \%$ gestiegen. In Deutschland wäre für einen längeren Zeitraum aufgrund des Nachholbedarfs aus den ersten zehn Jahren der WWU eine deutlich höhere Inflationsrate im zweiten Jahrzehnt notwendig und aufgrund der guten Konjunktur auch möglich gewesen. Tatsächlich belief sich hier der Anstieg der Lohnstückkosten jahresdurchschnittlich gerade einmal auf $2 \%$, die hausgemachte Inflation blieb mit durchschnittlich 1,5\% weiterhin deutlich unter der Zielmarke der EZB. ${ }^{37}$ Entscheidend für den Erfolg sind die simultane Betrachtung und Behandlung von Geldund Fiskalpolitik sowie Preis- und Lohnentwicklung in ih-

37 Vgl. European Commission: Forecast Autumn 2018. 
rer Wechselwirkung im makroökomischen Policy-Mix auf nationaler und Eurozonen-Ebene. ${ }^{38}$

In einer auch von der Sache her begründeten Subsidiarität kommt es hierbei zuerst auf die nationale Ebene an (ownership). In dem Maße, wie jeder Mitgliedstaat den Anforderungen der WWU - d. h. insbesondere der Goldenen Regel für Lohn- und Preisentwicklung, aber auch einem (erneut reformierten) SWP und einer (überarbeiteten) VMU - genügt (compliance), kann eine ergänzende oder korrigierende Rolle des Euroraums beschränkt bleiben. Je größer die Risikovermeidung in jedem Mitgliedstaat, desto geringer das verbleibende nationale „Rest-Risiko“, das im Euroraum z.B. in Gestalt einer makroökonomischen Stabilisierung geteilt werden muss. Alte und neue Instrumente der Economic Governance des Euroraums werden dann nur begrenzt in Anspruch genommen. Verbessert sich damit Effektivität und Effizienz dieser Instrumente, erhöht dies zugleich deren politische Akzeptanz.

Ein Makroökonomischer Dialog für jeden Mitgliedstaat und für den Euroraum sollte die „Arbeitsteilung“ zwischen den beiden Ebenen bei der Gestaltung der makroökonomischen Politikausrichtung und deren Institutionalisierung verbessern. Motivation hierfür sollte sein, dass alle beteiligten Akteure von einer Koordination der Wirtschaftspolitik durch Kooperation profitieren, ${ }^{39}$ insbesondere weil sie zum einen bei der Erreichung ihrer jeweils eigenen Ziele nicht autark, zum anderen in Bezug auf die Ziele der jeweils anderen Akteure nicht neutral sind. ${ }^{40}$

38 Es mangelt sicher nicht an einseitigen Signalen der makroökonomischen Akteure. Entscheidend ist, sie in Richtung eines konsistenten Policy-Mix zu lenken. Hierfür ist ein simultaner Dialog aller Beteiligten mit wechselseitiger Rückkopplung insbesondere in Krisenzeiten unentbehrlich.

39 „Several stakeholders would have the right incentives to act for the success of the package and stick to the agreement: wage earners and trade unions, for obvious reasons; governments, for additional fiscal income; and savers, for the prospect of interest rate normalisation. Finally, in a full employment context, employers have an incentive to implement wage increases to keep their best performing employees and, given that nominal labour costs of all employers would increase in parallel, they would (be) able to raise prices in line with the increase of their wage bills with limited risk of losing clients." Vgl. L. A. Pereira da Silva, B. Mojon, a.a. O., S. 2.

40 Beispiele hierfür sind eine geldpolitisch verursachte Stabilisierungsrezession oder eine lohnpolitisch induzierte Deflation - das bekannte „monetary management by trade unions“.

\section{Umsetzung}

Die Krise des Euroraums und noch verbliebene Anpassungsprobleme, aber vor allem die Vermeidung ihrer Wiederholung, die Wiedergewinnung einer nachhaltig stärkeren Dynamik und nicht zuletzt die Umsetzung des europäischen Grünen Deals auf der Basis einer tragfähigen makroökonomischen Politikausrichtung hat die EU-Kommission zu einer umfassenden „Economic governance review" veranlasst. ${ }^{41}$ Die gleichen Argumente sprechen für die Umsetzung der voranstehenden Vorschläge für die notwendige Erweiterung und Vertiefung der economic governance der WWU im Rahmen dieser Überprüfung.

Vor gut zwanzig Jahren hat die deutsche Präsidentschaft im Rahmen des Makroökonomischen Dialogs der EU erstmals alle Akteure an einen Tisch gebracht. Gleichzeitig hat sie dasselbe Format auf nationaler Ebene umgesetzt. Beide Gremien tragen seit zwanzig Jahren zur Konfliktvermeidung, Vertrauensbildung, gegenseitigem Verständnis und wertvollen Informationen für die Entscheidungsfindung jedes einzelnen Akteurs im Lichte des gemeinsamen Dialogs bei.

Im zweiten Halbjahr 2020 übernimmt Deutschland erneut die Ratspräsidentschaft. Deutschland sollte sie nutzen, nunmehr - wie vielfach vorgeschlagen und unterstützt von Notenbank und Sozialpartnern - die Einrichtung eines Makroökonomischen Dialogs für den Euroraum und für die Mitgliedstaaten nachdrücklich in die Beratung und Umsetzung der „Economic governance review“ einzubringen.

41 European Commission: Communication from the Commission to the European Parliament, the Council, the European Central Bank, the European Economic and Social Committee and the Committee of Regions: Economic Governance Review, Brüssel, 5.2. 2020. „Im Rahmen des Grünen Deals wird die Kommission den Prozess der makroökonomischen Koordinierung im Rahmen des Europäischen Semesters darauf ausrichten, die Ziele der Vereinten Nationen für nachhaltige Entwicklung darin einzubinden, ....“ Mitteilung der Kommission an das Europäische Parlament, den Europäischen Rat, den Rat, den Europäischen Wirtschafts-und Sozialausschuss und den Ausschuss der Regionen: Der Europäische Grüne Deal, S. 2.

Title: 20 Years of EU Macroeconomic Dialogue - A New Start for the Euro Area and Its Member States

Abstract: The EU Macroeconomic Dialogue came into existence 20 years ago. While overall macroeconomic development at the euro area-level has been satisfactory during its first decade, the massive macroeconomic divergences that have accumulated between member states, and the crisis it has caused, have highlighted the need for a deeper institutionalisation of macroeconomic coordination, in particular to take into account the need for greater coordination between the euro area and member states. To this end, a Macroeconomic Dialogue both at the euro area and at the national level has been established.

JEL Classification: E02, E61, F45 